\title{
Development of molecular markers for the identification of prolamins genes and their correlation with baking qualities of grain
}

\author{
Pirsikov A.S., Enzekrey E.S.*, Siksin S.V., Milyukova N.A. \\ All-Russia Research Institute of Agricultural Biotechnology, Moscow, Russia \\ * email: eynzeynkreyn@gmail.com
}

At the present time various molecular genetic markers are used for studies on biodiversity, phylogeny, and evolution. Storage proteins of cereals such as gliadins and glutenins are considered to be convenient markers of stable genes associations determinant baking qualities. That allows them to be used for solving practical problems. Effective molecular genetic markers have been developed for the identification of gliadin-encoding genes, that make it possible to establish the relationship of certain loci with the technological and nutritional qualities of triticale grain and significantly speed up the breeding process. The product amplification efficiency was confirmed by the presence of fragments of certain lengths and the absence of a PCR product in the negative control. According to literature data the size of the polyglutamine site play an important role in dough properties of triticale. The larger the polyglutamine site, the higher the quality of grain gluten, because large number of glutamine side chains is shown to increase visco-elasticity properties of dough. Based on the obtained results, it is impossible to make an definite conclusion of the amount of gliadins in a particular cultivar or line, since it is necessary to exclude pseudogenes by means of the cDNA syntesis. In the present study the bread-making quality analysis of diverse triticale genotypes was carried out. As a result three samples of spring triticale were isolated Lana, 131/714, and Grebeshok, which had the highest percentage of gluten content 21.3, 20.9 and 19.1\% respectively. Two of them (131/714; Grebeshok) in PCR analysis with primers Gli FII / Gli RII revealed an additional product. This fact requires further researchers.

Acknowledgments: The investigation was supported by the grant of the President of the Russian Federation No. MK-3235.2021.5. 